\title{
Primordial and late-time inflation in Brans-Dicke cosmology
}

\author{
M. Arık and M. C. Çalık
}

\begin{abstract}
The basic motivation of this work is to attempt to explain the rapid primordial inflation and the observed slow late-time inflation by using the BransDicke theory of gravity. We show that the ratio of these two inflation parameters is proportional to the square root of the Brans-Dicke parameter $\omega(\omega \gg 1)$. We also calculate the Hubble parameter $H$ and the time variation of the time dependent Newtonian gravitational constant $G$ for both regimes. The variation of the Hubble parameter predicted by Brans-Dicke cosmology is shown to be consistent with recent measurements: The value of $H$ in the late-time future is predicted as 0.86 times the present value of $H$.

Bog̃aziçi Univ., Dept. of Physics, Bebek, Istanbul, Turkey

E-mail: arikm@boun.edu.tr, cem.calik@boun.edu.tr
\end{abstract}

\section{Introduction}

The inflationary universe model whose key feature is a finite period of primordial rapid exponential expansion has been proposed to resolve a number of cosmological puzzles, including the horizon, flatness and monopole problems. In the original or 'old inflation model' [1], the universe super-cools into a false vacuum phase and its energy density acts as an effective cosmological constant which causes an epoch of de-Sitter (exponential) expansion. In this old inflation model, the de-Sitter expansion never ends and for a generic first order phase transition, there appears an energy barrier between the false vacuum and the true vacuum phases. This problem is known as the'graceful exit' problem. This problem was avoided with the invention of the new inflationary theory 2. In this theory, inflation may begin either in the false vacuum, or in an unstable state at the top of the effective potential. Then the inflaton field $\phi$ slowly rolls down to the minimum of its effective potential. The density perturbations produced during the slow-roll inflation are inversely proportional to $\dot{\phi}[3,4$. Thus the key difference between the new inflationary scenario and the old one is that the useful part of inflation in the new scenario, which is responsible for the homogeneity of our universe, does not occur in the false vacuum state, where $\dot{\phi}=0$. Although this scenario was so popular in the beginning of the 80 's, it had its own problems. One of them, for example, is that the inflaton field has an extremely small coupling constant in most versions of this scenario, so it could not be in thermal equilibrium with other matter fields. The theory 
of cosmological phase transitions, which was the basis of old and new inflation, did not work in this situation. Furthermore, inflation in this theory begins very late and during the preceding epoch the universe can easily collapse or become so inhomogeneous that inflation may never happen [5].

With the invention of the chaotic scenario all problems of old and new inflation were resolved. According to this scenario, inflation may occur even in theories with simple potentials such as $V(\phi) \sim \phi^{n}$. Inflation may begin even if there was no thermal equilibrium in the early universe, and it may start even at the Planckian density, in which case the problem of initial conditions for inflation can be easily resolved [5]. The field in this scenario evolved slowly and at this stage the energy density of the scalar field, unlike the density of ordinary matter, remained almost constant, and expansion of the universe continued with a much greater speed than in the old cosmological theory. Inflation does not require supercooling and tunnelling from the false vacuum [1], or rolling from an artificially flat top of the effective potential [2].

All models discussed so far have used field theories at very high energies to drive inflation. However, inflation may also be generated by changing the gravitational sector alone $\left(R^{2}\right.$ inflation) [6, 7] or both the gravitational and the matter sectors (Extended inflation) [8]. And the well known Jordan-Brans Dicke theories are widely used in this third type generation of inflation.

Jordan-Brans-Dicke theories are a class of theories in which the effective gravitational coupling evolves with time, and asymptotically attains a value of $G$. The strength of the coupling is determined by a scalar field, $\phi$, such that asymptotically, it tends to a value $G^{-1}$. The origins of Brans-Dicke theory is in Mach's principle according to which the property of inertia of material bodies arises because of their interactions with the matter distributed in the universe. In the modern context, the Brans-Dicke theory attempted to rescue the inflationary scenario from some of its problems. The theory is parameterized by a dimensionless constant $\omega$, where $\omega \rightarrow \infty$ as Brans-Dicke theory goes over to the Einstein theory 9]. Present limits of the constant $\omega$ based on time-delay [10, 11, 12, experiments require $\omega>500 \gg 1$. In the conventional inflationary scenario, the universe underwent an exponential expansion for a brief period in its early phase. After the exponential phase is over the universe should transit to the normal cosmology phase. Within the framework of Einstein-Hilbert action, there is no satisfactory mechanism by which the universe transits to the normal phase. It was shown that within the framework of Brans-Dicke gravity, a constant energy density leads to a rapid power-law expansion instead of exponential. This is rapid enough to solve the problems in standard cosmology and at the same time slow enough to make the transition to normal state possible after the inflationary phase. This has come to be known as extended inflation [13, 14. Extended inflation constrains $\omega$ to be less than 25. This bound comes from the fact that if it is more than 25 , there will be much more anisotropy in the Cosmic Microwave Background Radiation [15] than what is observed today. This is, however, incompatible with the bound which constrains $\omega$ to be greater than 500 [10, 12]. A large number of inflationary models were proposed in the framework 
of multi-scalar tensor gravity to solve the problem. For instance, the introduction of a potential for the scalar field $\phi$ and a scalar field dependent coupling constant $\omega(\phi)$ solved some problems ([16]-22]).

In our work, we start up with a strong link between inflation and Brans-Dicke 23. theory of gravity. The proposed model in this work is simple in that no other phenomenon, such as the domination of the false vacuum over the scalar field energy density as in the extended inflation model is used. Since the recent progress in observational cosmology shifted attention towards experimental verification of various inflationary theories, the motivation of this work is accelerated with the recent measurements of the dependence of the Hubble parameter $H=\frac{\dot{a}}{a}$ on the scale size $a(t)$ of the universe as

$$
\left(\frac{H}{H_{0}}\right)^{2}=\Omega_{\Lambda}+\Omega_{M}\left(\frac{a_{0}}{a}\right)^{3}
$$

where $\Omega_{\Lambda} \cong 0.75$ and $\Omega_{M} \cong 0.25$ [24]. In standard cosmology the $\Omega_{\Lambda}$ term would be induced by a cosmological constant. An immediate question which arises is the physical reason behind this cosmological constant. A universe, expanding under the sole influence of a cosmological constant $\Lambda=\lambda^{2}$ inflates as $a(t) \sim e^{\lambda t}$. For the present day expansion, $\lambda \simeq H_{0}$, whereas for the primordial expansion responsible for the present large size of the universe, $\lambda$ is much bigger.

In this paper, we will show a natural model where the large ratio of primordial inflation to present day inflation can be explained by Brans-Dicke theory which effectively replaces the Newtonian gravitational constant $G_{N}$ in the Einstein-Hilbert action by a power of the Brans-Dicke scalar field. The additional kinetic and potential terms of this scalar field in the action behave effectively as time dependent cosmological constants. We choose the kinetic term of the scalar field in the Lagrangian as $\frac{1}{2} g^{\mu \nu} \partial_{\mu} \phi \partial_{\nu} \phi$ in accordance with $(+---)$ metric signature. Then the length dimension of the scalar field $\phi$ in units where $c=\hbar=1$, is $L^{-1}$ so that $G_{N} \sim \phi^{-2} \sim L^{2}$. We make three simple assumptions: (1) The Brans-Dicke field $\phi$ does not couple to any other field except gravity. (2) The Lagrangian of the field, in addition to the kinetic term of $\phi$, contains the simplest chaotic inflation-style potential energy density $V(\phi)=\frac{1}{2} m^{2} \phi^{2}$ which is composed only of the scalar field mass term. (3) $\phi$ evolves with time.

The action is the following;

$$
S=\int d^{4} x \sqrt{g}\left[-\frac{1}{8 \omega} \phi^{2} R+\frac{1}{2} g^{\mu v} \partial_{\mu} \phi \partial_{\nu} \phi-V(\phi)+L_{M}\right]
$$

where $\phi$ represents the Brans-Dicke scalar field and $\omega$ denotes the dimensionless BransDicke parameter taken to be much larger than $1(\omega \gg 1) . L_{M}$, on the other hand, is the matter lagrangian such that the scalar field $\phi$ does not couple with it. $R$ is the Ricci scalar. For simplicity we also restrict our analysis to the Robertson Walker metric to emphasize that $\phi$ is necessarily spatially homogeneous;

$$
d s^{2}=d t^{2}-a^{2}(t) \frac{d \vec{x}^{2}}{\left[1+\frac{k}{4} \vec{x}^{2}\right]^{2}}
$$


where $k$ is the curvature parameter with $k=-1,0,1$ corresponding to open, flat, closed universes respectively and $a(t)$ is the scale factor of the universe.

After applying the variational procedure to the action and assuming $\phi=\phi(t)$ and energy momentum tensor of matter and radiation excluding $\phi$ is in the perfect fluid form of $T_{\nu}^{\mu}=\operatorname{diag}(\rho,-p,-p,-p)$ where $\rho$ is the energy density and $p$ is the pressure, and also noting that the right hand side of the $\phi$ equation below is set to be zero in accordance with our first assumption on $L_{M}$ being independent of $\phi$, the field equations reduce to (dot denotes $\frac{d}{d t}$ )

$$
\begin{aligned}
& \frac{3}{4 \omega} \phi^{2}\left(\frac{\dot{a}^{2}}{a^{2}}+\frac{k}{a^{2}}\right)-\frac{1}{2} \dot{\phi}^{2}-\frac{1}{2} m^{2} \phi^{2}+\frac{3}{2 \omega} \frac{\dot{a}}{a} \dot{\phi} \phi=\rho \\
& \frac{-1}{4 \omega} \phi^{2}\left(2 \frac{\ddot{a}}{a}+\frac{\dot{a}^{2}}{a^{2}}+\frac{k}{a^{2}}\right)-\frac{1}{\omega} \frac{\dot{a}}{a} \dot{\phi} \phi-\frac{1}{2 \omega} \ddot{\phi} \phi-\left(\frac{1}{2}+\frac{1}{2 \omega}\right) \dot{\phi}^{2}+\frac{1}{2} m^{2} \phi^{2}=p(5) \\
& \ddot{\phi}+3 \frac{\dot{a}}{a} \dot{\phi}+\left[m^{2}-\frac{3}{2 \omega}\left(\frac{\ddot{a}}{a}+\frac{\dot{a}^{2}}{a^{2}}+\frac{k}{a^{2}}\right)\right] \phi=0 .
\end{aligned}
$$

\section{Primordial inflation}

In the primordial inflation analysis, we start to solve the field equations (446) for an empty-static universe by setting $\dot{a}=0$ and $p=\rho=0$ and get the following vacuum solutions;

$$
\begin{aligned}
& \phi=\phi_{o} e^{\alpha t} \\
& a=a_{*}=\text { const } \\
& k=1 \text { (closed universe) }
\end{aligned}
$$

where

$$
\begin{aligned}
& \alpha^{2}=m^{2}\left(\frac{\omega}{2 \omega+3}\right) \\
& a_{*}^{2}=\frac{1}{m^{2}}\left(\frac{2 \omega+3}{3 \omega+3}\right)\left(\frac{3}{2 \omega}\right) .
\end{aligned}
$$

From these (7.9) solutions, we see that $\phi$ evolves exponentially with expansion parameter $\alpha$ and on the other hand $a_{*}$ is the constant size of this static universe. We regard that only the closed universe solution is possible as a positive aspect of this solution since homogeneity of the universe only makes sense if a closed universe undergoes big-bang. Let us note that, since two variables $\phi(t)$ and $a(t)$ satisfy the three equations (446), these solutions (7 8) are expected to be stable. To prove this stability we impose the size of the universe $a$ and the field $\phi$ to be a function of time $t$ as follows;

$$
\begin{aligned}
& a=a_{*}(1+\varepsilon b(t)) \\
& \phi=e^{\alpha t}(1+\varepsilon \psi(t))
\end{aligned}
$$


where $\varepsilon$ is the perturbation factor $(\varepsilon \ll 1)$ and $b(t), \psi(t)$ are perturbation functions of $a(t)$ and $\phi(t)$ respectively. We get the following differential equations by using (4,6) and equalities (10, 11)

$$
\begin{aligned}
& \dot{\psi}(t)-\frac{3}{2 \omega} \dot{b}(t)+\frac{3}{2 \omega \alpha a_{*}^{2}} b(t)=0 \\
& \ddot{\psi}(t)+(4+2 \omega) \alpha \dot{\psi}(t)+\ddot{b}(t)+2 \alpha \dot{b}(t)-\frac{b(t)}{a_{*}^{2}}=0 \\
& \ddot{\psi}(t)+2 \alpha \dot{\psi}(t)+3 \alpha \dot{b}(t)-\frac{3}{2 \omega} \ddot{b}+\frac{3}{\omega a_{*}^{2}} b=0 .
\end{aligned}
$$

Solving (14,16) simultaneously gives us that $\dot{b}=0$ is the only solution which implies $\ddot{b}=0$ and $\dot{\psi}=0$ and $\ddot{\psi}=0$. Namely, this closed universe vacuum solution where $a=a_{*}=$ cst and $\phi \sim e^{\alpha t}$ is stable.

We now investigate how the presence of radiation changes the behavior of the universe compared to this stable solution. Since solving the equations (4,6) for the primordial equation of state $p=\frac{1}{3} \rho$ is hard, we solve (6) for $a(t)$ by keeping $\phi \sim e^{\alpha t}$. By changing to the variable $a^{2}(t)=\theta(t)$, (6) turns out to be the following second order differential equation,

$$
-\frac{3}{4 \omega} \ddot{\theta}+\frac{3 \alpha}{2} \dot{\theta}+\left(\alpha^{2}+m^{2}\right) \theta=\frac{3}{2 \omega}
$$

with the following solution for $\theta(t)$,

$$
\theta(t)=a^{2}(t)=\left(\frac{3}{2 \omega}\right)\left(\frac{1}{\alpha^{2}+m^{2}}\right)+c_{1} e^{\beta_{1} t}+c_{2} e^{\beta_{2} t}
$$

where

$$
\beta_{1,2}=\frac{\frac{3 \alpha}{2} \pm \sqrt{\frac{9}{4} \alpha^{2}+\frac{3}{\omega}\left(\alpha^{2}+m^{2}\right)}}{\frac{3}{2 \omega}}
$$

and $\beta_{1}<0, \beta_{2}>0, c_{1}$ and $c_{2}$ are integration constants. Now, if we define the big-bang time as the limit when $t \rightarrow 0$ and $\omega \gg 1$, we get $\alpha \simeq \frac{m}{\sqrt{2}}, \beta_{1} \simeq-2 \alpha, \beta_{2} \simeq 2 \omega \alpha$. Equation (18) when expanded about $t=0$ becomes

$$
\theta(t)=a^{2}(t)=a_{*}^{2}\left(1+c_{1}+c_{2}-2 c_{1} \alpha t+2 c_{2} \alpha \omega t\right)
$$

with the constraint $1+c_{1}+c_{2}=0$ since we want $a^{2} \sim t$ as $t \rightarrow 0$. Thus, we end up with the general solution for the scale size of the universe in the primordial inflation regime with $\omega \gg 1$ as;

$$
a^{2}(t)=a_{*}^{2}\left[1-(1+c) e^{-2 \alpha t}+c e^{2 \alpha \omega t}\right] .
$$

This general solution is important for at least three reasons: (1) It is a natural solution in the sense that it does not need any special equation of state for the matter. It is solely deduced from the theory by using the stable-empty universe solution (7) in the equation (6). (2) When we examine this inflationary solution concerning as $t \rightarrow 0$ and as $t>0$ but not too much, we see that (21) is both consistent with $a(t) \sim \sqrt{t}$ as 
$t \rightarrow 0$ and also with primordial rapid inflation described by $a(t) \sim e^{\alpha \omega t}$ for $\omega \gg 1$. (3) We also check for $\omega \gg 1$ that if we substitute $\phi \sim e^{\alpha t}$ and $a \sim \sqrt{t}$ into (446) then the equation of state $p=\frac{1}{3} \rho$ is satisfied automatically as expected in this regime.

\section{Late-time inflation}

In this section, we analyze how much today's universe is far from late-time inflation by considering the case of slowly expanding empty universe $(\rho=p=0)$ except the $\phi$ field in it. Since the considered universe should be big, we ignore the curvature parameter $k / a^{2}$ as $a(t)$ increases with the expansion of the universe. Under these considerations, in analogy with the previous section, we put $a=e^{\tilde{\beta} t}$ and $\phi=e^{\tilde{\alpha} t}$ into (446) where $\tilde{\beta}, \tilde{\alpha}$ are new constants to be determined and search for a stable solution. We get the following coupled equations for $\tilde{\beta}$ and $\tilde{\alpha}$;

$$
\begin{aligned}
& \tilde{\beta}^{2}-\frac{2}{3} \omega \tilde{\alpha}^{2}+2 \tilde{\beta} \tilde{\alpha}-\frac{2 \omega}{3} m^{2}=0 \\
& \tilde{\beta}^{2}+\left(\frac{2}{3} \omega+\frac{4}{3}\right) \tilde{\alpha}^{2}+\frac{4}{3} \tilde{\beta} \tilde{\alpha}-\frac{2 \omega}{3} m^{2}=0 \\
& \tilde{\beta}^{2}-\frac{\omega}{3} \tilde{\alpha}^{2}-\omega \tilde{\beta} \tilde{\alpha}-\frac{\omega}{3} m^{2}=0 .
\end{aligned}
$$

These equations have the solution;

$$
\begin{aligned}
& \tilde{\beta}=2(\omega+1)\left(\frac{\omega}{6 \omega^{2}+17 \omega+12}\right)^{1 / 2} m \approx 0.8 \sqrt{\omega} m \\
& \tilde{\alpha}=\left(\frac{\omega}{6 \omega^{2}+17 \omega+12}\right)^{1 / 2} m \approx \frac{0.4}{\sqrt{\omega}} m
\end{aligned}
$$

where the approximations are again for $\omega \gg 1$ so that $m \approx \sqrt{2} \alpha$.

We see that although the primordial inflation parameter is $0.7 \omega m$, the late-time inflation parameter is found to be $0.8 \sqrt{\omega} m$. Namely, a factor $\sqrt{\omega}$ less than the primordial inflation parameter. This is a very important result in the sense that although there is an experimental lower bound on $\omega$, there is no upper bound [10] on it, hence in Brans-Dicke cosmology, the late-time inflation can be as small as one wishes compared to the primordial inflation.

Now, we consider the case where the universe is closed $(k=1)$ and matter dominated $p \approx 0$. Since solving the field equations (446) for $a(t)$ and $\phi(t)$ under $p \approx 0$ is hard enough, we proceed to work by defining the rate of change in $\phi$ as $F(a)=\dot{\phi} / \phi$ and Hubble parameter as $H(a)=\dot{a} / a$, and rewriting the right hand side of the field equations(446) in terms of $H, F$ and their derivatives with respect to $a$ (prime denotes $\left.\frac{d}{d a}\right)$

$$
\begin{aligned}
& H^{2}-\frac{2 \omega}{3} F^{2}+2 H F+\frac{1}{a^{2}}-\frac{2 \omega}{3} m^{2}=\left(\frac{4 \omega}{3}\right) \frac{\rho}{\phi^{2}} \\
& H^{2}+\left(\frac{2 \omega}{3}+\frac{4}{3}\right) F^{2}+\frac{4}{3} H F+\frac{2 a}{3}(H \dot{H}+H \dot{F})+\frac{1}{3 a^{2}}-\frac{2 \omega}{3} m^{2}=\left(\frac{-4 \omega}{3}\right) \frac{p}{\phi^{2}} \approx 0
\end{aligned}
$$


Primordial and late-time inflation in Brans-Dicke cosmology

$$
H^{2}-\frac{\omega}{3} F^{2}-\omega H F+a\left(\frac{H \dot{H}}{2}-\frac{\omega}{3} H \dot{F}\right)+\frac{1}{2 a^{2}}-\frac{\omega}{3} m^{2}=0 .
$$

Expanding $F(a)$ and $H(a)$ in powers of $\left(\frac{a_{o}}{a}\right)$ up to third order, where $a_{0}$ is the present size of a universe,

$$
\begin{aligned}
& H(a)=H_{\infty}+H_{2}\left(\frac{a_{0}}{a}\right)^{2}+H_{3}\left(\frac{a_{0}}{a}\right)^{3} \\
& F(a)=F_{\infty}+F_{2}\left(\frac{a_{0}}{a}\right)^{2}+F_{3}\left(\frac{a_{0}}{a}\right)^{3}
\end{aligned}
$$

and putting them into (27, 29), we get the perturbation constants defined above for $(\omega \gg 1)$ :

$$
\begin{aligned}
& H_{\infty}=\tilde{\beta} \approx 0.8 \sqrt{\omega} m \\
& H_{2} \approx-\frac{1}{2 a_{o}^{2} H_{\infty}} \approx-\frac{0.6}{\sqrt{\omega} a_{o}^{2} m} \approx 0 \\
& F_{\infty}=\tilde{\alpha} \approx \frac{0.4}{\sqrt{\omega}} m \\
& F_{2} \approx \frac{3}{4 \omega a_{o}^{2} H_{\infty}} \approx \frac{0.9}{\omega^{3 / 2} a_{o}^{2} m} \approx 0 \\
& H_{3} \approx \frac{2 \omega}{3} F_{3} \\
& H_{3} \approx-F_{3} .
\end{aligned}
$$

Up to now, we note that all the constants required in our assumption for $H(a)$ and $F(a)$ in the late-inflation regime are almost determined from the theory except $H_{3}$ and $F_{3}$. Indeed solving equations (36) and (37) simultaneously gives us $H_{3}$ and $F_{3}$ to be zero. But to explain the late-time universe we may assume that $\frac{p}{\rho} \ll 1$ rather than $p$ being exactly zero. To overcome this problem, we use the relation (36) between $H_{3}$ and $F_{3}$ coming from the equation (29) which is more exact comparing to the relation (37) coming from the equation (28). We also use the classical Friedman formula which is used for fitting observations of Hubble parameter to density parameter $\Omega$;

$$
\frac{H^{2}}{H_{0}^{2}}=\Omega_{\Lambda}+\Omega_{R}\left(\frac{a_{0}}{a}\right)^{2}+\Omega_{M}\left(\frac{a_{0}}{a}\right)^{3}
$$

where $\Omega_{\Lambda}$ is the vacuum density parameter, $\Omega_{R}$ is the curvature density parameter, $\Omega_{M}$ is the matter density parameter, and $H_{0}$ is the present Hubble parameter. Using (32, [33), we rearrange (30) leaving $H_{3}$ as a free parameter and put into (38) to get

$$
\begin{aligned}
& \Omega_{\Lambda} \approx \frac{H_{\infty}^{2}}{H_{0}^{2}} \\
& \Omega_{R} \approx \frac{2 H_{\infty} H_{2}}{H_{0}^{2}}
\end{aligned}
$$




$$
\Omega_{M} \approx \frac{2 H_{\infty} H_{3}}{H_{0}^{2}}
$$

Using the present observational result [24] $\Omega_{M} \approx 0.25, \Omega_{\Lambda} \approx 0.75$ and $\Omega_{R} \approx 0$, we find $H_{3}$ and $F_{3}$ to be,

$$
\begin{aligned}
& H_{3} \approx 0.13 \sqrt{\omega} m \quad(\omega \gg 1) \\
& F_{3} \approx \frac{1.41}{\sqrt{\omega}} m(\omega \gg 1) .
\end{aligned}
$$

After finding the perturbation constants explicitly for $H$ and $F$, we also find it worthy to determine how the Hubble parameter $H(a)=\dot{a} / a$ and the time variation of $G$, where $G$ is the time dependent value of the gravitational constant, change in the primordial and late-time regimes compared to their present values. To do so, we use the fact that since Brans-Dicke gravity becomes identical to Einstein gravity as $\omega$ approaches infinity, the kinetic term for the scalar field $\frac{1}{8 \omega} \phi^{2}$ in the action (2) will be the same as that of the term $1 / 16 \pi G$ in the Hilbert-Einstein action. Using this fact we get the relation between the scalar field $\phi$ and $G$ as

$$
G^{-1}=\frac{2 \pi \phi^{2}}{\omega}
$$

Then, putting equations (32), (33), (42) into (30) for the present value of the Hubble constant $H_{0}$ and for the present value of the scale factor of the universe $a_{0}$, gives us the magnitude of expansion parameter $\alpha$, and mass $m$ of the scalar field when $\omega \gg 1$ as,

$$
\begin{aligned}
& \alpha \approx \frac{0.70}{\sqrt{\omega}} H_{0} \\
& m \approx \frac{1.08}{\sqrt{\omega}} H_{0} .
\end{aligned}
$$

Similarly, using (44) and putting equations (34), (35), (43) into (31) for the present value of the scale factor of the universe $a=a_{0}$, gives us the magnitude of the present value of the parameter $\dot{G} / G$ when $\omega \gg 1$ as,

$$
\left(\frac{\dot{G}}{G}\right)_{0} \approx-\frac{1.81}{\sqrt{\omega}} m \approx-\frac{1.95}{\sqrt{\omega}} H_{0} .
$$

On the other hand, since $\phi \approx e^{0.7 m t}, a \approx e^{0.7 m \omega t}$ and $\phi \approx e^{\frac{0.4}{\sqrt{\omega}} m t}, a \approx e^{0.8 \sqrt{\omega} m t}$ in the primordial and late-time regimes respectively, using (44) we get the parameter $\dot{G} / G$ and the Hubble parameter $H=\dot{a} / a$ in these regimes as

$$
\begin{aligned}
& \left(\frac{\dot{G}}{G}\right)_{\text {primordial }} \approx-1.4 m \approx-\frac{1.51}{\sqrt{\omega}} H_{0} \\
& \left(\frac{\dot{G}}{G}\right)_{\text {late-time }} \approx \frac{-0.81}{\sqrt{\omega}} m \approx-\frac{0.88}{\omega} H_{0} \\
& (H)_{\text {primordial }} \approx 0.7 m \omega \approx 0.75 \sqrt{\omega} H_{0}
\end{aligned}
$$




$$
(H)_{\text {late-time }} \approx 0.8 \sqrt{\omega} m \approx 0.86 H_{0} .
$$

Lastly, we investigate the ratio $\nu=\frac{p}{\rho}$ where $p$ and $\rho$ are pressure and energy density of the late-time universe respectively as $\omega \rightarrow \infty$

$$
\nu=\frac{p}{\rho}=\frac{-\frac{\omega+6}{\omega(6 \omega+6)} a_{0}^{-2}-H_{\infty} H_{3} \frac{20 \omega+21}{\omega(3 \omega+3)}}{\frac{\omega \omega+3}{\omega(2 \omega+2)} a_{0}^{-2}+2 H_{\infty} H_{3}} \simeq 0 \quad(\omega \gg 1)
$$

and find it approaching a value of zero as expected.

\section{Discussion and Conclusion}

In this work we have investigated the nature of the simplest chaotic inflation-style potential energy density $V(\phi)=\frac{1}{2} m^{2} \phi^{2}$ which is composed only of the scalar field mass term. We have assumed that and $\phi$ evolves with time in Brans-Dicke Cosmology with a perfect fluid distribution. We have found a general solution in exponential form for the size of the universe in primordial regime inflating with an expansion parameter $\omega \alpha$. We also calculated how the Hubble parameter $H(a)=\dot{a} / a$ and the time variation of $G$, where $G$ is the time dependent value of the gravitational constant, change in the primordial and late-time regimes compared to their present values. We note that the newest measurement [24] of $\Omega_{\Lambda}$ and $\Omega_{M}$ has been used as input to derive these results. One interesting feature is that the predicted present day and primordial values of $|\dot{G} / G|$ are comparable whereas the asymptotic value is much smaller. In any case a measurement of $\dot{G} / G$ will be crucial in determining the Brans-Dicke parameter $\omega$. On the other hand, the Hubble parameters predicted by the theory in both regimes have

yielded interesting results. Besides this, the ratio $\nu=\frac{p}{\rho}$ is found to be zero as universe approaches late-time inflation $(\omega \gg 1)$.

In the end we can say that the ratio of the primordial and late-time inflation parameters is proportional to $\sqrt{\omega}$ is the most appealing feature of Brans-Dicke cosmology. Thus, recent measurements, which imply that in today's universe $\Omega_{\Lambda} \neq 0$, require $1 / \omega \neq 0$ and make this model attractive.

\section{Acknowledgments}

We would like to thank the anonymous referee for thoughtful comments and valuable suggestions on this paper.

\section{References}

[1] A. H. Guth, Inflationary Universe: A possible solution to the horizon and flatness problems, 1981 Phys. Rev. D 23347.

[2] A. D. Linde, A New Inflationary Scenario: A possible solution of the Horizon, Flatness, Homogeneity, Isotropy, and Primordial Monopole Problems, 1982 Phys. Lett. B 108 389; A. Albrecht and P. J. Steinhardt, Cosmology for Grand Unified Theories with Radiatively Induced Symmetry Breaking, 1982 Phys. Rev. Lett. 481220. 
[3] V. F. Mukhanov and G. V. Chibisov, Quantum Fluctuations and Nonsingular Universe, 1981 JETP Lett. 33 532; V. F. Mukhanov, Gravitational Instability of the Universe filled with a Scalar Field, 1985 JETP Lett. 41493.

[4] S. W. Hawking, The development of irregularities in a Single Bubble Inflationary Universe, 1982 Phys. Lett. B 115 295; A. H. Guth and S. Y. Pi, Fluctuations in the New Inflationary Universe, 1982 Phys. Rev. Lett. 491110.

[5] A. D. Linde, Particle Physics and Inflationary Cosmology (Harwood, Chur, Switzerland, 1990).

[6] F. S. Acceta, D. J. Zoller, and M. S. Turner, Induced-gravity inflation, 1985 Phys. Rev. D 31 3046; S. Barr and G. Segre, Limits on Reheating Inflationary Cosmology Models, 1989 Phys. Rev. Lett. 62 2781; Inflation and reheating in induced-gravity models, 1990 Phys. Rev. D 412398.

[7] B. L. Spokoiny, Inflation and generation of perturbations in broken-symmetric theory of gravity, 1984 Phys. Lett. 147B 39; R. Fakir and W. G. Unruh, Improvment on cosmological chaotic inflation through nonminimal coupling, 1990 Phys. Rev. D 411783.

[8] D. La, P. J. Steinhardt, and E. W. Bertschinger, Prescription for successful extended inflation, 1989 Phys. Lett. B 231 231; E. J. Weinberg, Some problems with extended inflation, 1989 Phys. Rev. D 403950.

[9] C. M. Will, The confrontation between General Relativity and Experiment: An update, 1984 Phys. Rep.113 345.

[10] R. D. Reasenberg et al., Viking Relativity Experiment: Verification of signal retardation by solar gravity, 1961 Astrophys. J. 234925.

[11] C. M. Will, Theory and Experiment in Gravitational Physics (Cambridge University Press, 1993), revised ed; p. 123; in Proceedings of the 1998 Slac Summer Institude on particle physics (gr-qc/ 9811036).

[12] E. Gaztanaga and J. A. Lobo, Nonlinear Gravitational Growth of Large-Scale Structures Inside and Outside Standard Cosmology, 2001 Astrophys. J. 54847.

[13] D. La and P. J. Steinhardt, Extended Inflationary Cosmology, 1989 Phys. Rev. Lett. 62376.

[14] C. Mathiazhagan and V.B. Johri, An inflationary universe in Brans-Dicke Theory: a hopeful sign of theoretical estimation of the gravitational constant, 1984 Class. Quantum. Gravity. 129.

[15] P. de Bernardis et al; First results from the BOOMERanG experiment, 2000 Nature 400955.

[16] A. L. Berkin, K. Maeda and J. Yokohoma, Soft inflation, 1991 Pyhs. Rev. Lett. 65 141; A. L. Berkin and K. Maeda, Inflation in generalized Einstein theories, 1991 Phys.Rev. D 441691.

[17] R. Holman, E. W. Kolb, S. L. Vadas and Y. Wang, Scale-invariant extended inflation, 1991 Phys. Rev. D 433833.

[18] T. Damour, G. W. Gibbons and C.Gundlach, Dark matter, time-varying G, and a dilaton field, 1990 Phys. Rev. Lett. 64 123; T. Damour and C. Gundlach, Nucleosynthesis constraints on an extended Jordan-Brans-Dicke theory, 1991 Phys. Rev. D 433873.

[19] P. J. Steinhardt and F. S. Accetta, Hyperextended inflation, 1990 Phys. Rev. Lett. 642740.

[20] A. Green and A. Liddle, Conditions for successful extended inflation, 1996 Phys.Rev. D 542557.

[21] D. La, Inflationary cosmology based on Weyl-scaled induced gravity, 1991 Phys. Rev. D 441680.

[22] T. Chiba, Generality of inflation and constraints on scalar-tensor theories of gravity, 1997 Class. Quantum. Grav. 142951.

[23] C. Brans and C. H. Dicke, Mach's Principle and a Relativistic Theory of Gravitation, 1961 Phys. Rev. 124925.

[24] R. A. Knop et al., New Constraints on $\Omega_{\mathrm{M}}, \Omega_{\Lambda}$, and $\omega$ from an Independent Set of Eleven HighRedshift Supernovae Observed with HST, 2003 ApJ. 598102. 